\title{
Publisher Correction: Pandemic data challenges
}

Correction to: Nature Machine Intelligence https://doi.org/10.1038/s42256-020-0172-7, published online 8 April 2020.

An earlier version of this Editorial provided inaccurate information about the accessibility of COVID-19 related content provided by Springer Nature. Springer Nature is making all COVID-19 related content free to access. This Editorial has been corrected to reflect this.

Published online: 27 April 2020

https://doi.org/10.1038/s42256-020-0179-0

๑) The Author(s), under exclusive licence to Springer Nature Limited 2020 